\title{
Lumen
}

Selected Proceedings from the Canadian Society for Eighteenth-Century Studies

\section{Les limites de l'hospitalité et la géographie suisse : l'exemple de Julie ou La Nouvelle Héloïse de Jean-Jacques Rousseau}

\section{Antonio Viselli}

Volume 31, 2012

URI : https://id.erudit.org/iderudit/1013076ar

DOI : https://doi.org/10.7202/1013076ar

Aller au sommaire du numéro

Éditeur(s)

Canadian Society for Eighteenth-Century Studies / Société canadienne d'étude du dix-huitième siècle

ISSN

1209-3696 (imprimé)

1927-8284 (numérique)

Découvrir la revue

Citer cet article

Viselli, A. (2012). Les limites de l'hospitalité et la géographie suisse : l'exemple de Julie ou La Nouvelle Héloïse de Jean-Jacques Rousseau. Lumen, 31, 183-196. https://doi.org/10.7202/1013076ar

Copyright (c) Canadian Society for Eighteenth-Century Studies / Sociéte canadienne d'étude du dix-huitième siècle, 2012
Ce document est protégé par la loi sur le droit d'auteur. L'utilisation des services d'Érudit (y compris la reproduction) est assujettie à sa politique d'utilisation que vous pouvez consulter en ligne.

https://apropos.erudit.org/fr/usagers/politique-dutilisation/ 


\title{
Les limites de l'hospitalité et la géographie suisse : l'exemple de Julie ou La Nouvelle Héloïse de Jean-Jacques Rousseau
}

\author{
Antonio Viselli \\ University of Toronto
}

C'est l'affluence des hôtes qui détruit l'hospitalité1.

Penser l'altérité est à la base du fondement de la socialisation et de la subjectivité moderne. Dans le cadre de l'intersubjectivité, l'autre, le tout Autre est à la fois celui contre qui le «je » se confronte et se définit, ainsi que celui qui dévoile l'autre qui est en soi. L'hospitalité s'inscrit dans ce discours philosophique qu'est l'altérité, cependant délimitant les frontières et le lieu d'accueil ou de transgression: le «chez soi », la propriété dite privée, l'extérieur de l'intérieur. Accueillir un invité, accommoder un passant, voyageur ou vagabond, accepter l'intrus: voici les circonstances de l'hospitalité et voici également les possibilités qui permettent d'interroger le processus de la socialisation des êtres selon les écrits de Rousseau. À la lumière de la théorie sur l'hospitalité selon Derrida, ce travail, prenant comme point de départ la «Lettre XXIII » dans Julie ou La Nouvelle Héloïse, se veut à la fois transtextuel, intertextuel et intratextuel, combinant le Don Quichotte de Cervantès revisité par Rousseau et la théorie du social dans d'autres textes de Rousseau. C'est par le truchement d'un dialogue synchronique entre les œuvres mêmes de Rousseau, en particulier avec Les dialogues, et d'une conversation diachronique avec Derrida et la

1. Jean-Jacques Rousseau, Emile ou De l'éducation, dans Euvres complètes, vol. 4, édition publiée sous la direction de Bernard Gagnebin et Marcel Raymond, Paris, Gallimard, 1969, p. 774. 
critique subséquente, que l'on analysera la manifestation de l'hospitalité dans la «Lettre XXIII» et l'intérêt de repenser la sociabilité de l'être selon ce paradigme.

\section{Rousseau et l'hospitalité: un exemplum du picaresque}

Dans sa forme la plus courante, l'hospitalité se définit encore au XVIII ${ }^{\mathrm{e}}$ siècle comme l'action propre à recevoir chez soi un invité ou un inconnu, à le nourrir et à le loger gratuitement. Les conditions de l'hospitalité ont évolué depuis l'Antiquité, époque où elles représentaient le «[d]roit réciproque pour ceux qui voyageaient de trouver, selon des conventions établies entre des particuliers, des familles, des villes, gîte de protection les uns chez les autres²». Au Moyen-Âge et dans les romans de chevalerie, l'hospitalité respecte ces conventions où l'hôte, qui se bat au nom de Dieu et pour l'honneur d'un peuple, reçoit des habitants des lieux de passage l'hospitalité, ce qui leur permet de s'acquitter ainsi d'une obligation morale envers le chevalier. Lon pourrait même parler de loi de l'hospitalité. Cela expliquerait l'étonnement d'un Don Quichotte nourri des romans de la Table ronde, face à l'obligation de payer sa nuit dans une pension. Cette définition semble marquer de manière implicite que l'hospitalité est un pacte résultant de la manipulation de certains signes, une xenia telle que décrite par Derrida, où l'hôte propose les conditions de l'accueil, tout en gardant sa souveraineté de maître 3 . L'ambigüité polysémique fait que le terme «hôte » peut faire référence à la fois à celui qui invite et à celui qui est invité ou reçu. Alors, il semblerait, d'un point de vue étymologique, qu'une altérité innée soit le fondement de l'hospitalité, où l'autre se confronte au soi, aussi bien qu'à l'autre de soi au sein du soi. Selon le mot de Peter Melville: «Accueillir l'autre sans préavis signifie, surtout, accueillir l'arrivée de l'autre au sein de soimême, un autre qui permet au soi de voir au-delà de soi ${ }^{4}$.» Être maître

2. Trésor de la langue française informatisé, [En ligne. Portail lexical du Centre National de Ressources Textuelles et Lexicales] http ://www.cnrtl.fr/definition/hospitalité (21 février 2010).

3. Les citations de Jacques Derrida sont reprises des notes de séminaire d'Anne Dufourmantelle, dans Anne Dufourmantelle, Jacques Derrida à répondre de l'hospitalité, Paris, Calmann-Lévy, 1997, p. 45.

4. Peter Melville, Romantic Hospitality and the Resistance to Accommodation, Waterloo, Wilfried Laurier UP, 2007, p. 33. C'est moi qui traduis. 
de soi et du chez-soi, accepter l'étranger ou l'inconnu sans préavis problématise la conception d'hospitalité chez Rousseau qui, rarement hôte lui-même, était souvent reçu par ses amis, les Montmorency, par exemple, comme le montrent ses confidences à Malesherbes ou même ses textes les plus acclamés tels que Julie ou La Nouvelle Héloïse, ou les plus contestés ou censurés comme Émile.

\section{Hospitalité ou persécution?}

Si le terme «hospitalité », loin d'être commenté par l'auteur, n'apparaît que dans trois textes de Rousseau, notamment dans Les Confessions, dans Julie ou La Nouvelle Héloïse et dans l'Émile, il suffit de lire une biographie sur Jean-Jacques Rousseau pour comprendre la place qu'occupent l'accueil et l'hébergement dans son œuvre sans toutefois oublier que le rejet, le refus, voire la condamnation par contumace de l'homme, de l'auteur et de ses œuvres en représentent nécessairement la face antithétique: continuellement ballotté entre deux extrêmes à la limite de l'indéfinissable, seule la fuite devient l'unique constante. Inextricablement liée à cette fuite est la manie d'un paranoïaque avili par un sentiment de persécution perpétuel.

Or, sans trop s'attarder sur des faits contingents, il serait toutefois souhaitable de combler des lacunes dans la biographie de Rousseau en s'interrogeant davantage sur le rôle de l'hospitalité dans la vie de l'auteur. Bien que Barthes ait proclamé la mort de l'auteur, Rousseau reste bien vivant et présent dans ses textes, opérant sans cesse une distanciation et un rapprochement vis-à-vis de ses personnages français, suisse, espagnol ou autre. Il est donc parfois possible de remplacer un personnage fictif par la figure de l'auteur lui-même et une lecture paradigmatique se montre souvent fructueuse, substitution à faire cependant avec précaution, surtout dans la «Lettre XXIII ».

\section{La «Lettre XXII» : le haut et le bas Valaisan}

Afin d'éviter la tension et les possibles spéculations familiales sur la relation amoureuse entre Julie et son tuteur Saint-Preux, l'héroïne envoie ce dernier entreprendre un voyage d'affaires. La « Lettre XXIII » narre ce voyage dans le bas et le haut Valaisan de Saint-Preux. Elle évoque des spectacles naturels qui l'émerveillent et le «zele hospitalier» des habitants du Vaud, rappelons-le, la terre de Madame de 
Warens 5 . Rousseau peint un tableau du contexte naturel qui prépare, par le biais de la désorientation, un mélange de cadres spatio-temporels qui suggèrent l'union entre un présent nouveau et un passé idéal, un monde si proche et en même temps si lointain:

[C]es pays étranges si bizarrement contrastés; la nature sembloit encore prendre plaisir à s'y mettre en opposition avec elle-même, tant on la trouvait différente en un même lieu sous divers aspects. Au levant les fleurs du printems, au midi les fruits de l'automne, au nord les glaces de l'hiver: elle réunissoit toutes les saisons dans le même instant, tous les climats dans le même lieu, des terrains contraires sur le même sol, et formoit l'accord inconnu par tout ailleurs des productions des plaines et de celles des Alpes ${ }^{6}$.

Cette lettre se structure selon une série d'opposition qui prépare la dichotomie entre le haut et le bas Valaisan aussi bien que l'hospitalité conditionnelle et inconditionnelle, la première opposition étant l'intériorité psychologique de Saint-Preux face à l'extérieur naturel :

Je voulais rêver, et j’en étois toujours détourné par quelque spectacle inattendu. Tantôt d'immenses roches pendoient en ruines au dessus de ma tête. Tantôt de hautes et bruyantes cascades m'inondoient de leur épais brouillard. Tantôt un torrent éternel ouvrait à mes côtés un abîme dont les yeux n'osoient sonder la profondeur [...] Un mélange étonnant de la nature sauvage et de la nature cultivée, montroit par tout la main des hommes, où l'on eut cru qu'ils n'avoient jamais pénétré $[. . .]^{7}$

Le paysage provoque un choc intérieur à l'observateur qui se sent quasiment menacé par une nature inconnue et terrible, où abondent précipices et dangers, une nature sublime au sens kantien. L'effet que produit la nature sur l'âme de l'observateur provoque avant tout une peur ou un sentiment de grandeur qui le rend conscient de sa propre petitesse. Ensuite, le calme s'instaure et la relation entre sujet et objet se redéfinit; de l'âme de l'objet inanimé naturel émane l'aura qui permet une sorte d'épiphanie chez l'individu. La force de la nature est telle qu'elle repousse donc les frontières dans la psyché de l'être aussi bien qu'entre l'être humain et son environnement naturel: elle

5. Jean-Jacques Rousseau, Julie ou La Nouvelle Héloïse, dans Euvres complètes, vol. 2, op. cit., 1969, p. 79 .

6. Ibid., p. 77.

7. Ibid., p. 77. 
repousse constamment les seuils et les limites (sub-limen) du cadre et du soi à l'égard de l'Autre.

Saint-Preux renchérit sur la nature qu'il tente de décrire par sa plume: «Imaginez la variété, la grandeur, la beauté de mille étonnans spectacles; le plaisir de ne voir autour de soi que des objets tout nouveaux, des oiseaux étranges, des plantes bizarres et inconnues, d'observer en quelque sorte une autre nature, et de se trouver dans un nouveau monde ${ }^{8}$. Comme le rappellent Henri Coulet et Bernard Guyon, cette évocation souligne chez le héros «[l]e besoin de l'“inconnu", du "nouveau" qui s’allient intimement à celui de la pureté, de la virginité, de l'innocence, du paradis terrestre. Ainsi le "nouveau monde" est un des aspects que peut revêtir cet "autre monde" où vivent les héros du roman"». Suite aux observations des deux critiques, un fort parallélisme peut s'établir entre le contexte de la «Lettre XXIII » et celui du monde idéal dépeint dans les Dialogues, au point où il serait légitime de se demander, en tenant compte de l'anachronisme, si ce pays du Vaud ne représenterait pas les ruines avant la lettre du monde idéal délabré des Dialogues. Les «habitants du monde enchanté ${ }^{10}$ » vivent dans le milieu suivant:

Figurez-vous donc un monde idéal semblable au nôtre, et néanmoins tout différent. La nature y est la même que sur notre terre, mais l'economie en est plus sensible, l'ordre en est plus marqué, le spectacle plus admirable; les formes sont plus élégantes, les couleurs plus vives, les odeurs plus suaves, tous les objets plus interessans ${ }^{11}$.

Le monde idéal, autrement appelé le monde des initiés, permet de rapprocher le contexte naturel de la socialisation, d'une élévation morale, car l'initié est celui qui est reçu ou que l'on admet à connaître des mystères, à dévoiler des secrets. Le sublime épiphanique de l'initiation souligne cette transformation interne par le biais de l'externe, s'alignant avec la pensée de Rousseau sur la religion naturelle telle que décrite dans «La profession de foi du Vicaire Savoyard» et de connivence avec les préceptes de l'hospitalité aussi.

\footnotetext{
8. Ibid., p. 79 .

9. Ibid., p. 1391. p. 672 .

10. Jean-Jacques Rousseau, Les Dialogues, dans (Euvres complètes, vol. 1, op. cit., 11. Ibid., p. 668.
} 


\section{L'hospitalité vaudoise}

Le Valaisan, plongé entre un passé indéfini et un présent spectaculaire, spectateur dans un véritable théâtre de la nature qui reflète la dynamique du corps et de l'âme, se définit à la fois par l'horizontalité de la prairie et la verticalité des Alpes: «Trà l'erba verde e’l bel monte vicino/ Levan di terra al Ciel nostr'intelletto ${ }^{12}{ }^{2}$. Ces vers de Pétrarque notés par Rousseau traduisent bien la poésie et l'intelligence d'une telle peinture. C'est ainsi que la géométrie naturelle prépare la dichotomie hospitalière des hauts et bas Valaisans. Ces derniers, contrairement aux hauts Valaisans, rançonnent

[...] assés durement les passagers, et j'avois peine à concilier dans un même peuple des manieres si différentes, écrit Saint-Preux. Un Valaisan m’en expliqua la raison. Dans la vallée, me dit-il, les étrangers qui passent sont des marchands, et d'autres gens uniquement occupés de leur négoce et de leur gain. Il est juste qu'ils nous laissent une partie de leur profit et nous les traitons comme ils traitent les autres ${ }^{13}$.

La convenance et le profit règnent dans cet échange condescendant entre habitants et marchands: il s'agit bien d'une véritable économie de l'hospitalité, voire, d'une anti-hospitalité par rapport au signe traditionnel mais porte-parole assez loquace de notre industrie touristique d'aujourd'hui.

Toutefois, si les bas Valaisans restent bas, terrestres et excessivement matérialistes, il n'en est pas autant des habitants du haut Valais. Selon ce mot de Dedeyan, «à l'ascension physique va correspondre pour Jean-Jacques Saint-Preux l'ascension morale ${ }^{14} »$. Saint-Preux - qu'il ne faudrait pas trop rapidement assimiler à Rousseau tel que le fait Dedeyan - narre son étonnement face à l'accueil de ce peuple:

Mais ce que je n'ai pu vous peindre et qu'on ne peut guère imaginer, c'est leur humanité désintéressée, et leur zele hospitalier pour tous les étrangers que le hazard ou la curiosité conduisent chez eux. [... ] Quand j'arrivois le soir dans un hameau, chacun venoit avec tant d'empresse-

12. «[...] les hêtres s'élancent de l'herbe au sommet des monts et semblent élever au ciel avec leurs têtes, les yeux et l'esprit des mortels. » Traduction libre de Rousseau lui-même, (Julie ou La Nouvelle Héloüse, p. 79; p. 1391 pour la traduction).

13. Ibid., p. 80.

14. Charles Dedeyan, La Nouvelle Héloïse de Jean-Jacques Rousseau: étude d'ensemble, Paris, Société d'édition d'enseignement supérieur, 1990, p. 35. 
ment m’offrir sa maison que j'étois embarrassé du choix, et celui qui obtenoit la préférence en paroissoit si content que la première fois je pris cette ardeur pour de l'avidité. Mais je fus bien étonné quand après en avoir usé chez mon hôte à peu près comme au cabaret, il refusa le lendemain mon argent, s'offensant même de ma proposition, et il en a par tout été de même. Ainsi c'étoit le pur amour de l'hospitalité, [... ] qu'à sa vivacité j’avais pris pour l'âpreté du gain [... ]. En effet à quoi dépenser de l'argent dans un pays où les maîtres ne reçoivent point le prix de leurs fraix, ni les domestiques celui de leurs soins, et où l'on ne trouve aucun mendiant ${ }^{15}$ ?

S'ils s'enrichissaient, ils finiraient eux aussi par se comporter comme les habitants du bas-Valais et voici deux possibilités antithétiques auxquelles Saint-Preux, apportant avec lui un bagage culturel et juridique qui masque la bonté comme l'avidité, et conscient des pactes implicites des échanges sociaux, doit faire face. La frontière entre invité et hôte, le lieu d'échange et d'accueil sous-tendent l'espace neutre, espace où la souveraineté dominante et la force du pouvoir n'a pas lieu au sens qu'attache Derrida au terme. C'est ainsi que s'exprime SaintPreux:

Ce qui me paroissoit le plus agréable dans leur accueil, c'étoit de n'y pas trouver le moindre vestige de gêne ni pour eux ni pour moi. Ils vivoient dans leur maison comme si je n'y eusse pas été, et il ne tenoit qu'à moi d'y être comme si j’y eusse été seul. Ils ne connoissent point l'incomode vanité d'en faire les honneurs aux étrangers, comme pour les avertir de la présence d'un maître, dont on dépend au moins en cela. Si je ne disois rien, ils supposoient que je voulois vivre à leur maniere; je n'avois qu'à dire un mot pour vivre à la mienne, sans éprouver jamais de leur part la moindre marque de répugnance ou d'étonnement. [...] Ils en usent entre eux avec la même simplicité; les enfans en âge de raison sont les égaux de leurs pères, les domestiques s'asseyent à table avec leurs mâ̂tres; la même liberté regne dans les maisons et dans la république, et la famille est l'image de l'Etat ${ }^{16}$.

Alors que le juste-milieu ne rend pas tout à fait justice à la cause, Rousseau semble mettre en valeur un compromis entre l'homme naturel et l'homme social. Dans Julie ou La Nouvelle Héloïse, roman qui précède le Contrat social d'une année, l'auteur est déjà capable de

15. Jean-Jacques Rousseau, Julie ou La Nouvelle Héloïse, op. cit., p. 80.

16. Ibid., p. 81. 
voir le fonctionnement de ses théories abstraites en formes concrètes. Le micro chez Rousseau contient toujours le macro. Métonymiquement l'infiniment grand rentre dans l'infiniment petit: l'État, la Nation se fonde sur l'unité de base sociale, le couple et la famille. Dans Du contrat social, Rousseau écrira : «La plus ancienne de toutes les sociétés et la seule naturelle est celle de la famille [...] [elle] est donc si l'on veut le premier modèle des sociétés politiques; le chef est l'image du père, le peuple est l'image des enfans, et tous étant nés égaux et libres n'aliénent leur liberté que pour leur utilitée ${ }^{17}$ ». Il est vrai que pour le philosophe du préromantisme, l'individu est inaliénable, sa liberté n'appartient qu'à lui seul. Cependant, il existe des cas où sa liberté se cache entre silence et moquerie, où sa liberté est prise en otage par l'intérieur.

\section{Un texte parasitique: La «lettre XXIII » et Don Quichotte}

Bien qu'heureux et touché par l'accueil des locaux, Saint Preux s'avoue gêné par certaines mœurs: «la durée excessive des repas» et « rester une partie de la journée et boire d'autant» à table ${ }^{18}$. Se taisant malgré son malaise, Saint Preux se compare à un des premiers héros du roman moderne:

Pour moi qui respecte encore plus les usages des pays où je vis que ceux de la galanterie, je recevois leur service en silence, avec autant de gravité que D. Quichotte chez la Duchesse. J'opposois quelquefois en souriant les grandes barbes et l'air grossier des convives au teint éblouissant de ces jeunes beautés timides, qu'un mot faisoit rougir, et ne rendoit que plus agréables ${ }^{19}$.

D'un point de vue intertextuel, Don Quichotte de Cervantès relève d'un texte parasitique, l'exemple d'une hospitalité biologique forcée qui ronge la «Lettre XXIII ». Cependant, il s'agit bien d'une construction minutieuse de la part d'un auteur qui avait d'abord bien lu le texte de Cervantès et qui a su davantage le transformer selon ses propres besoins. La référence au Quichotte pourrait passer inaperçue mais cette simple comparaison a son importance: elle rapproche l'état

17. Jean-Jacques Rousseau, Du Contrat social, dans Euvres complètes, vol. 3, op. cit., p. 35 .

18. Jean-Jacques Rousseau, Julie ou La Nouvelle Hélö̈se, op. cit., p. 82.

19. Ibid., 82 . 
convivial de Saint Preux de celui du Quichotte qui, lui aussi, en route vers un monde d'aventures idéalisé par le roman chevaleresque, n'est que de passage entre une terre et une autre, une aventure et de nouveaux exploits, entre la réalité et l'utopie. L'accueil hospitalier que représente cette transition intermédiaire et éphémère d'un être en flux constant, s'élargit au-delà de l'accueil privé et domestique pour s'intégrer dans un nouveau monde, dans une nouvelle hiérarchie sociale. En passant, Rousseau mentionne l'épisode de la duchesse, l'interprétant cependant avec subtilité et nuance, ce qui nous permet de lire, au moins en partie, Don Quichotte à travers la perception de Rousseau.

L'histoire intercalée de Don Quichotte et de la duchesse fait suite à la mésaventure «Le bateau enchanté » où Sancho et Don Quichotte, risquant de se noyer, sont sauvés par des meuniers à qui appartenait la barque. De loin, le chevalier errant perçoit une dame vêtue de vert tenant dans sa main gauche un oiseau rapace de mauvais augure, signe présageant le sinistre à venir. Don Quichotte envoie son écuyer parler avec la gente dame qui, les reconnaissant d'après les aventures qu'elle avait lues, insiste pour qu'ils demeurent chez elle. Il est important de noter que nous sommes dans la deuxième partie du roman, la première ayant été lue par une grande partie des personnages qui apparaissent dans la seconde, ajoutant une couche métatextuelle à la fiction, voire une conscience de leur propre fictionnalité au sein d'une œuvre. Selon l'ethos chevaleresque, Don Quichotte accepte ce geste de bonté qu'est l'invitation, lui qui vit selon les lois de la parcimonie, se nourrissant de baies dans la nature et dormant sur du foin à la belle étoile, contrairement à Sancho qui préfère les repas copieux, où le vin coule à flots et les mets recouvrent entièrement la table. Une fois installé chez le duc et la duchesse, Sancho Panza ne cesse de raconter des anecdotes qui embarrassent son maître, en particulier à cause de l'insistance de la part du duc à ce que Don Quichotte, l'invité s'assoie à la tête de la table, à la cabezera. Sancho, en démasquant l'hypocrisie de la courtoisie traditionnelle, raconte naturellement l'histoire d'un autre hidalgo qui demande à son invité de prendre sa place symbolique à table: "Sentaos, Majagranzas, que adondequiera que yo me siente será vuestra cabecera $^{20} »$. Le tissu hiérarchique se défait autour de la table qui, ronde

20. "Asseyez-vous nigauds, quelle que soit la place que j'occuperai, elle sera toujours à la tête de votre table» (C'est moi qui traduis). Miguel de Cervantes, Don 
ou carrée, est un lieu de rencontre et d'échange. La duchesse oblige Sancho et son maître à se faire raser et coiffer à table par les servantes et ce, avec l'eau de la vaisselle, acte d'effémination et d'assujettissement puisque la barbe représente la virilité aussi bien que le savoir et l'expérience. La barbe, symbole de cohésion au sein d'une maison à travers la métaphore du patriarcat, devient un signe ridicule d'autodérision. Saint-Preux qui observe l'opposition entre les barbus et le teint des jolies demoiselles fait allusion à cette cohésion qui risque de se démanteler et qu'il ne réussit point à comprendre. Il est possible, par le biais d'une autre substitution paradigmatique, d'imaginer à la place du Quichotte, Rousseau qui se croirait l'objet de moquerie de ceux qui l'entourent, en particulier plus tard de Hume et ses amis. Son complexe de persécution est sans doute le détail qui l'amènera à modifier le texte de Cervantès.

\section{Saint-Preux, Don Quichotte et les Valaisans}

Saint-Preux subit son dérangement «en silence» et «avec autant de gravité que D. Quichotte chez la duchesse ${ }^{21} »$. Cependant, dans le roman de Cervantès, Don Quichotte ne subit point les fléaux chez la duchesse avec "gravité", car, d'un point de vue sémiotique, il ne lit pas leur accueil comme un manque d'hospitalité. Bien au contraire, ignorant leur jeu, il est tellement touché par la forme et la cérémonie qui éclosent du contexte, qu'il est convaincu une fois pour toutes qu'il est un véritable chevalier errant. Rousseau, en l'interprétant ainsi, enlève Don Quichotte du contexte de l'enchantement et le rend conscient de ses alentours, du jeu et de la fausse hospitalité alors que c'est exclusivement cette galanterie qui convainc le chevalier de son identité dans Don Quichotte. Cette lecture élèverait le statut du Quichotte à une figure christique qui souffre qu'on se moque de lui. Cette représentation du protagoniste se fait au détriment de l'herméneutique du roman de Cervantès, mais elle trouve sa place lorsqu'adaptée selon les besoins de Rousseau. Saint-Preux incarnerait alors un juste milieu entre Don Quichotte et Sancho Panza, avec qui il partage plus que les mêmes initiales. C'est lui le véritable personnage qui comprend

Quijote de la Mancha, édition de. F. Sevilla, Madrid: Alianza editorial, Biblioteca Cervantes, 2001, II, 31, p. 281.

21. Jean-Jacques Rousseau, Julie ou La Nouvelle Hélö̈se, op. cit., p. 82. 
- au moins en partie - la machination de la société, qui apprend la texture d'une hiérarchie obsolète et corrompue. Rappelons-le, Sancho Panza est un paysan (et donc plus proche de la nature et de la terre qu'il laboure), mais qui, selon Rousseau, rentre dans l'engrenage du progrès, car l'être humain dès qu'il commence à légitimer la multiplicité des lois qui régissent la société dont il fait partie ne peut arrêter son désir de tout gouverner. C'est justement le cas de Sancho qui finira par gouverner l'île qu'il a tant désirée.

Comparer Saint-Preux à Don Quichotte alors que celui-là souligne son dégoût pour la galanterie témoigne d'un autre renversement tactique de la part de Rousseau, ce qui lui permet de renchérir sur son horreur pour les raffinements mondains. Davantage, si l'homme part de l'état naturel, c'est par le truchement de la rationalité qu'il applique des lois à ses états subséquents, au fur et à mesure que l'humanité évolue. Or, plus le législateur promulgue des lois, plus la division sociale devient remarquable; le travail en est aussi affecté suivant la même équation. Selon ce mot de Starobinski, «[1]e mal ne réside pas dans la nature humaine, mais dans les structures sociales» et ce sont la pompe et la cérémonie, la forme ornementale et superflue qui brouillent les sens ${ }^{22}$. C'est bien le cas pour Saint-Preux qui se trouve incapable et même saisi d'étonnement et de surprise face à une nature en parfaite harmonie avec des êtres humains. Les propos déistes auxquels adhérait Rousseau permutent le texte, révélant le croisement entre une religion naturelle et une religion sociale. Tout comme la cérémonie, la pompe et le rituel chez Rousseau exposent l'incongruité entre la religion interne de l'individu (religion naturelle) et la foi proclamée en masse à travers le culte d'adoration commun, la forme que prend cette cérémonie superficielle demeure l'unique différentiation hiérarchique entre l'aristocratie, un bourgeois et un paysan. Au-delà de ces catégories discriminatoires, la contrepartie à ces formalités ridicules se recrée à partir de son plus petit dénominateur commun, la famille: «les enfans en âge de raison sont les égaux de leurs pères, les domestiques s'asseyent à table avec leurs maîtres ${ }^{23}$ ". Bien que Rousseau ne semble pas se déclarer ouvertement contre la hiérarchisation de la société, croyant avant tout à la perfectibilité de l'être

22. Jean Starobinski, La transparence et l'obstacle, Paris, Gallimard, 1971, p. 26.

23. Jean-Jacques Rousseau, Julie ou La Nouvelle Héloüse, op. cit., p. 81. 
humain, c'est la légitimation du pouvoir de celui qui gouverne qui importe.

\section{À la lumière de Derrida: l'hospitalité inconditionnelle}

La «Lettre XXIII » prépare un milieu naturel propice à ce que Derrida appelle l'hospitalité inconditionnelle. Si l'hospitalité telle que nous la concevons se définit selon une xenia, un pacte et un telos, c'est-à-dire un échange entre hôte (souverain) et hôte (accueilli) avec des règles préétablies, alors l'hospitalité inconditionnelle existerait hors des contraintes d'un pacte, sans convenances. Derrida soutient que, bien que d'emblée impossible, cette hospitalité absolue est nécessaire et représente donc le but auquel il faut aspirer. Les circonstances propices à cet idéal requièrent l'acte de donner à l'autre, à l'étranger, à l'invité, même à l'intrus tout sans poser de questions, sans imposer de restrictions, sans demander de récompenses, une hospitalité inconditionnelle et sans souveraineté24. Pour Derrida, "l'hospitalité absolue exige que j'ouvre mon chez-moi et que je donne non seulement à l'étranger [... ] mais à l'autre absolu, inconnu, anonyme et que je lui donne lieu, que je le laisse venir, que je le laisse arriver, et avoir lieu dans le lieu que je lui offre, sans lui demander ni réciprocité ni même son nom », même si cet étranger bouleverse mon chez-moi, ma souveraineté, même s'il me prend en otage ${ }^{25}$.

Alors que Derrida pense l'hospitalité et par extension l'altérité selon la politique nationale actuelle, en l'occurrence celle de la France, il est possible de rapprocher cette vision de celle de Rousseau. Lacte de «donner lieu» comme l'explique Derrida s'étend à ce que pourrait signifier pour Rousseau un second état de Nature. Le premier état de Nature, auquel il faudrait remonter d'un point de vue ethnocentrique et que les stéréotypes rousseauistes prétendent récupérer, se convertirait néanmoins en deuxième état de Nature. À ce moment là, les frontières se dissoudraient et l'hospitalité idéale deviendrait en quelque sorte la cause de son impossibilité, car la demeure et la propriété privée s'annuleraient. Westmoreland dans son article «Interruptions: Derrida and Hospitality» explique que les lois de l'hospitalité sont liées à l'État

24. Mark W. Westmoreland, «Interruptions: Derrida and Hospitality», Kritike, 2, 1 , juin 2008, p. 3.

25. Anne Dufourmantelle, op. cit., p. 29. 
souverain, c'est-à-dire à ce qui détermine les lois et les devoirs. L'hospitalité absolue (ou inconditionnelle), cependant, exige ce qui va audelà du devoir et du droit; elle fait fi du droit, devenant une « loi sans loi» et voici justement la distinction entre « une loi inconditionnelle d'un désir absolu pour l'hospitalité et une loi, une politique, une éthique conditionnelles» libres de subordination ou de dettes ${ }^{26}$. L'ipse se défait de sa sécurité, de son autorité, de sa propriété. Le signe maison se dissout et les limites du dedans et du dehors demeurent incertaines: l'invité devient l'hôte (l'accueillant) et l'hôte l'invité dans un milieu qui n'est plus son chez-soi. À ce moment-là, la division entre recevant et reçu se brouille, l'espace de l'hospitalité se neutralisant, entraîne la déconstruction du «chez-soi». Ainsi cette problématique pourrait-elle se reformuler en ces termes: «L'hospitalité absolue ne peut exister de manière illimitée qu'en dehors des paramètres de la loi et des concepts. Les conditions d'une telle hospitalité sont à la fois les conditions de sa possibilité et de son impossibilité27 ». Il s'agit donc à la fois d'un problème dans le fondement théorique de Derrida et dans la conception du pouvoir chez Rousseau. Car, bien que renversée, la relation entre hôte et hôte n'en est jamais une entre pairs: l'un aura toujours un pouvoir sur l'autre, alors que le droit au pouvoir n'existe pas. Chez Rousseau, les êtres humains sont nés égaux et ce sont les lois qui pervertissent l'état naturel.

\section{Conclusion}

Qu'il s'agisse d'un roman épistolaire, d'un traité philosophique, d'un roman autobiographique ou autres, nous retrouvons parsemés dans ces quelques textes des bribes, voire le véritable fondement d'une pensée sur le social, sur l'altérité, sur la famille et sur l'individu. Cette analyse de la manifestation de l'hospitalité dans le texte de Rousseau, bien que celui-ci ne théorise pas sur le sujet explicitement, permet au critique du $\mathrm{XXI}^{\mathrm{e}}$ siècle de tisser des liens entre le concept d'état naturel, de notion de religion civile et naturelle, aussi bien que de la conception d'une utopie sociale telle que décrite dans le «monde idéal» des Dialogues. Jean-Jacques Rousseau, lui-même étranger, parfois double-

26. Mark W. Westmoreland, op. cit., p. 4. C'est moi qui traduis.

27. Ibid., p. 4. 
ment étranger par exemple quand il écrit le Discours sur l'origine et les fondements de l'inégalité parmi les hommes, il connaît bien l'état de xenos et par extension, la xenia ou le pacte qui accompagne l'accueil chez autrui, sous forme de visite ou à la recherche d'un asile. Alors qu'il n'est pas souhaitable de substituer où bon il nous semble le nom de Rousseau à celui de ses personnages, il est vrai que ses récits rappellent des moments de sa vie non négligeables. En conséquence, il est possible de voir comment l'auteur de Julie ou la Nouvelle Héloïse a pu être tenté de prendre la place de Saint-Preux, aussi bien que celle évoquée dans le rôle du Quichotte qu'il interprète et remanie à son gré. L'intrus textuel au sein de la «Lettre XXIII » permet d'expliciter davantage le ridicule de la pompe et de la cérémonie, de l'arbitraire des divisions sociales et du manque de légitimité dans l'ordre des causes. Cependant, le contexte naturel et sublime du Vaud crée l'idéal qu'aurait pu revêtir le «nouveau monde »-semblable à l'hospitalité absolue, antérieure au droit et donc au chez-soi - réel potentiel d'une tabula rasa. Il s'agit d'un monde à la fois projeté vers le passé inconnu qui n’a sans doute jamais existé, vers un avenir hypothétique et qui n’aura sans doute jamais lieu: compromis spatio-temporel entre l'homme civil et l'homme naturel, lieu fertile et germe potentiel d'un second état de Nature. 